\title{
Mode-of-action and evolution of methylenedioxy bridge forming P450s in plant specialized metabolism
}

\author{
Akio Noguchi ${ }^{1}$, Manabu Horikawa ${ }^{2}$, Jun Murata ${ }^{2}$, Masayuki Tera ${ }^{2}$, Yosuke Kawai ${ }^{3}$, \\ Masaji Ishiguro ${ }^{4}$, Toshiaki Umezawa ${ }^{5}$, Masaharu Mizutani ${ }^{6}$, Eiichiro Ono ${ }^{1, *}$ \\ ${ }^{1}$ Research Institute, Suntory Global Innovation Center Ltd., Mishima, Osaka 618-8503, Japan; ${ }^{2}$ Bioorganic Research Institute, \\ Suntory Foundation for Life Sciences, Mishima, Osaka 618-8503, Japan; ${ }^{3}$ Department of Integrative Genomics, Tohoku \\ Medical Megabank Organization, Tohoku University, Sendai, Miyagi 980-8575, Japan; ${ }^{4}$ Niigata University of Pharmacy and \\ Applied Life Sciences, Niigata Niigata 956-8503, Japan; ${ }^{5}$ Research Institute for Sustainable Humanosphere, Kyoto University, \\ Uji, Kyoto 611-0011, Japan; ${ }^{6}$ Graduate School of Agricultural Science, Kobe University, Kobe, Hyogo 657-8501, Japan \\ *E-mail: bcj06215@nifty.com Tel: +81-75-962-2244 Fax: +81-75-962-3791
}

Received July 6, 2014; accepted August 28, 2014 (Edited by T. Koeduka)

\begin{abstract}
Sesamin is a major furofran-class lignan in sesame seeds and harbors characteristic two methylenedioxy bridges (MDB) that are sequentially formed from (+)-pinoresinol via (+)-piperitol by a Sesamum indicum P450, CYP81Q1. However, the molecular basis for this unique catalytic activity of CYP81Q1 has been poorly understood. To elucidate MDB formation, we tested various natural and non-natural metabolites as substrates for CYP81Q1. A synthetic $(+)-S C 1 \mathrm{mr}$ and a naturally occurring (+)-kobusin showed inhibitory effect on the production of (+)-sesamin by CYP81Q1 unlike $(+)$-epipinoresinol and (-)-pinoresinol, indicating the strict diastereomer and enantiomer selectivity. Homology modeling followed by site-directed mutagenesis of CYP81Q1 showed that an amino acid residue crucial for MDB formation is a unique Ala residue (A308), located in I-helix proximal to the substrate pocket, responsible to the conserved distal-Thr residue. MDB by CYP81Q1 is produced possibly through the formation of a substrate-participated hydrogen-bonding network, since single replacement of the Ala by Thr severely and specifically lowered the MDB forming activity. This hypothesis is supported by a newly identified MDB-forming enzyme CYP81Q38 from Phryma leptostachya harboring an Ala responsible to Ala308 in CYP81Q1. An evolutional perspective of CYP81Q1 is discussed in relation to another MDBforming CYP719As functionally conserved in Ranunculales.
\end{abstract}

Key words: P450, lignan, methylenedioxy bridge, lamiales, convergent evolution.

Lignans are a class of plant specialized metabolites that are elaborated from phenylpropanoid (C6-C3 unit; e.g., coniferyl alcohol) dimers widespread in the plant kingdom (Umezawa 2003a, 2003b), and have been shown to exhibit a diverse array of biological activities in mammals, as exemplified by those in anti-oxidative, anti-tumor, anti-mitotic and anti-viral properties (Apers et al. 2003; Ayres and Loike 1990; MacRaw and Towers 1984). While lignans attract rapidly growing interests from pharmaceutical and biotechnological viewpoints for their effects on human health, their physiological roles in planta have been poorly understood (Satake et al. 2013).

Sesame (Lamiales, Pedaliaceae, Sesamum indicum L.) is a major annual oilseed crop that produces a large amount of lignans represented by $(+)$-sesamin and $(+)$-sesaminol glucosides with two characteristic methylenedioxy bridges (MDB) on the aromatic rings (Katsuzaki et al. 1992, 1994; Moazzami and Kamal-Eldin 2006; Noguchi et al. 2008; Osawa et al. 1985). MDB structure is often observed in naturally occurring specialized metabolites in various plant lineages, some of which are used for traditional natural medicines and plant resources for drugs and spices (Figure 1). For instance, stylopine in California poppy (Eschscholzia californica), noscapine in opium poppy (Papaver somniferum), and berberine in Coptis japonica belong to a group of isoquinoline alkaloids observed in Ranunculales plants. MDB can also be found in other classes of alkaloids including lycorine in Lycoris radiata and piperine in pepper (Piper nigrum). The occurrence of specialized metabolites with MDB is not limited to alkaloids: isoflavones pisatin in pea (Pisum sativum) and

Abbreviations: CYP, cytochrome P450; MDB, Methylenedioxy bridge; PSS, piperitol/seamin synthase; SDS-PAGE, sodium dodecyl sulfatepolyacrylamide gel electrophoresis; $\mathrm{CBaB}$, coomassie brilliant blue; HPLC, high performance liquid chromatography; LC-IT-TOF-MS, liquid chromatography ion trap time-of-flight mass spectrometry; RT-PCR, reverse transcription-polymerase chain reaction; GAPDH, glyceraldehyde 3-phosphate dehydrogenase; (+)-Prmr, (+)-pinoresinol methylether reverse; (+)-SC1 mr, (+)-sesamin catechol 1 methylether reverse.

This article can be found at http://www.jspcmb.jp/

Published online December 10, 2014 

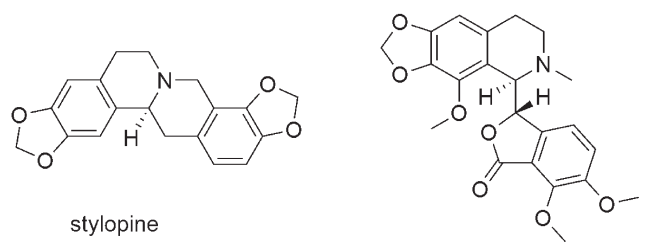

$$
\text { noscapine }
$$<smiles></smiles>

lycorine<smiles>COc1ccc2c(c1)OC[C@@]1(O)c3cc4c(cc3O[C@H]21)OCO4</smiles>

pisatin<smiles>COc1cc([C@H]2c3cc4c(cc3[C@@H](O)[C@@H]3COC(=O)[C@H]23)OCO4)cc(OC)c1OC</smiles>

podophyllotoxin<smiles>COc1cc(CC2C(=O)OCC2Cc2ccc3c(c2)OCO3)cc(OC)c1OC</smiles>

yatein<smiles></smiles>

berberine<smiles>O=C1OC[C@H](Cc2ccc3c(c2)OCO3)C1Cc1ccc2c(c1)OCO2</smiles>

hinokinin

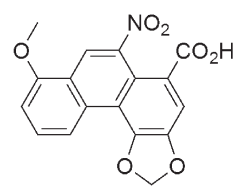

Aristolochic acid<smiles>C=CCc1ccc2c(c1)OCO2</smiles>

safrole

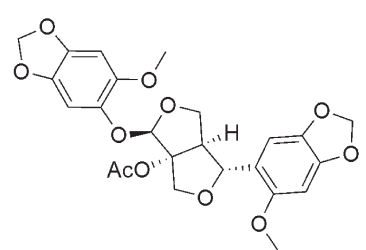

Phrymarolin I<smiles>O=c1c(-c2ccc3c(c2)OCO3)coc2cc(O)ccc12</smiles>

pseudobaptigenin<smiles>OC[C@@H](Cc1ccc2c(c1)OCO2)[C@H](CO)Cc1ccc2c(c1)OCO2</smiles>

dihydrocubebin<smiles>C=CCc1cc(OC)c2c(c1OC)OCO2</smiles>

apiole

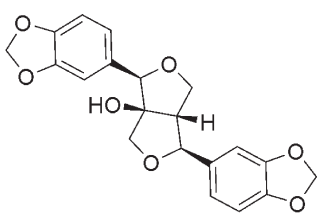

Paulownin

Figure 1. Naturally occurring specialized metabolites with MDB structure. Stylopine in California poppy (Eschscholzia californica), noscapine in opium poppy (Papaver somniferum), Berberine in Coptis japonica, lycorine in Lycoris radiata, piperine in pepper (Piper nigrumis), pisatin in pea (Pisum sativum), pseudobaptigenin in chickpea (Cicer arietinum), podophyllotoxin in Podophyllum hexandrum, yatein in Anthriscus sylvestris, dihydrocubebin in Piper cubeba, hinokinin in Linum corymbulosum, safrole in Sassafras officinale, apiole in parsley (Petroselinum crispum), aristolochic acid in Asarum sieboldii, phrymarolin I in Phryma leptostachya, paulownin in Paulownia tomentosa.

peudobaptigenin in chickpea (Cicer arietinum), volatile phenylpropenes safrole in Sassafras officinale and apiole in parsley (Petroselinum crispum). Podophyllotoxin in Podophyllum hexandrum, yatein in Anthriscus sylvestris, dihydrocubebin in Piper cubeba and hinokinin in Chamaecyparis obtuse and Linum corymbulosum are examples of lignans harboring MDB moiety. In addition, aristolochic acid in Asarum sieboldii is known as a unique aromatic carboxylic acid harboring a MDB.

Oxidative formation of MDB moiety is catalyzed by two discrete classes of P450 monooxygenases (P450s). Previously we identified CYP81Q1 from S. indicum as a piperitol/sesamin synthase (PSS) that catalyzes two sequential MDB formations on (+)-pinoresinol, yielding $(+)$-sesamin via $(+)$-piperitol (Figure 2) (Ono et al. 2006). Moreover CYP81Q2, CYP81Q3 and CYP81Q4 were isolated from $S$. radiatum, $S$. alatum, and $S$. schinzianum, respectively, showing that CYP81Q genes are structurally conserved among Sesamum genus.

CYP719A enzymes are another class of MDBforming P450 for isoquinoline alkaloids (Ikezawa et al. 2003, 2007, 2009; Takemura et al. 2013; Winzer et al. 2012). More recently, CYP719A23 and CYP719A24 from Podophyllum spp. were shown to catalyze MDB formation from (-)-matairesinol to (-)-pluviatolide (Marques et al. 2013). Important to note, MDB formation is a unique type of oxygenation reaction because of the lack of oxygen derived from P450 in the products. In spite of general occurrence of MDB as a chemical structure observed in an array of specialized metabolites, molecular mechanism of MDB formation by $\mathrm{P} 450$ has been poorly understood.

In this report, we characterized MDB formation mechanism, 1) with selected lignans which inhibit MDB 


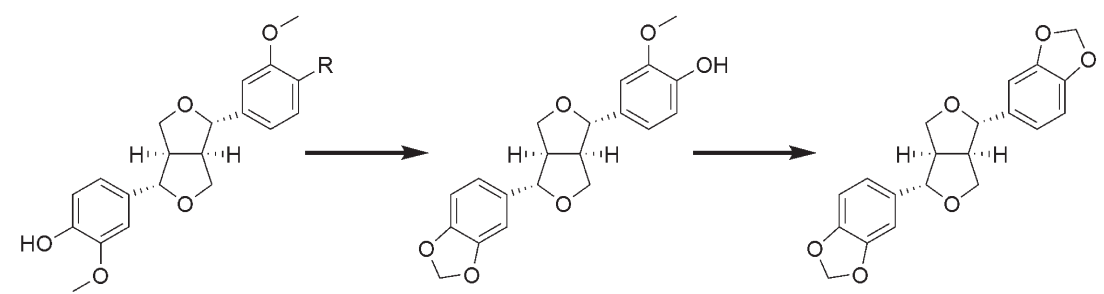

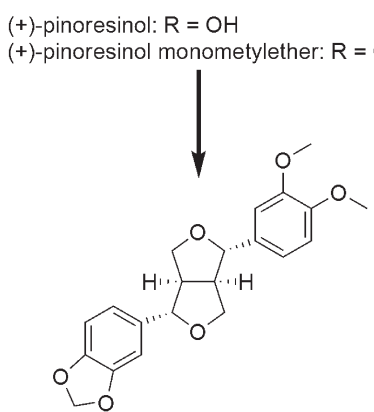

(+)-kobusin

(+)-piperitol<smiles>COc1cc(C2OCC3COCC3C2c2ccc(O)c(OC)c2)ccc1O</smiles>

(+)-epipinoresinol<smiles>COc1ccc(C2CC3C(CO2)COC3c2ccc(OC)c(OC)c2)cc1OC</smiles>

(+)-phillygenin<smiles>COc1cc(C2OC[C@@H]3[C@H]2CO[C@H]3c2ccc(O)c(OC)c2)ccc1O</smiles>

(-)-pinoresinol<smiles>COc1cc(OCC2COCC2c2ccc3c(c2)OCO3)ccc1O</smiles>

(+)-sesamolinol<smiles>OCOc1cc2ccc1COCC1C(Oc3ccc4c(c3)OCO4)OCC21</smiles>

(+)-sesamolin<smiles>[R]c1ccc([C@H]2OC[C@@H]3[C@@H](c4ccc(OC)c(O)c4)OC[C@@H]23)cc1[R]</smiles>

(+)-Prmr1: $\mathrm{R}^{1}=\mathrm{OH}, \mathrm{R}^{2}=\mathrm{OCH}_{3}$ (+)-Prmr2: $\mathrm{R}^{1}=\mathrm{OCH}_{3}, \mathrm{R}^{2}=\mathrm{OH}$<smiles>[R]c1ccc([C@@H]2OC[C@H]3[C@@H](c4ccc5c(c4)OCO5)OC[C@@H]32)cc1O</smiles>

(+)-SC1: $\mathrm{R}=\mathrm{OH}$ (+)-SC1mr: $\mathrm{R}=\mathrm{OCH}_{3}$<smiles>Oc1ccc([C@H]2OC[C@@H]3[C@H](c4ccc(O)c(O)c4)OC[C@H]23)cc1O</smiles>

$(+)-S C 2$<smiles>COc1cc(C2OCC3COCC3CO2)cc(OC)c1O</smiles>

(+)-syringaresinol<smiles>COc1cc(CC2COC(c3ccc(O)c(OC)c3)C2CO)ccc1O</smiles>

(+)-lariciresino<smiles>COc1cc(CC2COC(=O)C2Cc2ccc(O)c(OC)c2)ccc1O</smiles>

(-)-matairesinol

Figure 2. Chemical structures of lignans. Horizontal arrow indicates MDB formation by CYP81Q1 from $(+)$-pinoresinol to $(+)$-sesamin via (+)-pipiritol (Ono et al. 2006). Vertical arrow indicates MDB formation by CYP81Q1 from (+)-pinoresinol monomethylether to (+)-kobusin (This work).

formation on (+)-piperitol by CYP81Q1, and 2) by site-directed mutagenesis upon CYP81Q1 based on the homology models. Through a series of investigations, we propose a possible scheme for MDB formation. We also discuss an evolutional perspective of MDB forming enzymes in lineage-specific specialized metabolisms with identification of a novel PSS member, CYP81Q38 from Phryma leptostachya, in comparison to CYP719As found in Ranunculales.

\section{Materials and methods}

\section{Chemicals}

Two sesamin metabolites, $(1 R, 2 S, 5 R, 6 S)-6$-(3,4-dihydroxyphenyl)-2-(3,4-methylenedioxyphenyl)-3,7-dioxabicyclo[3,3,0]octane $((+)$-SC1) and $(1 R, 2 S, 5 R, 6 S)$-2,6-bis(3,4-dihydroxyphenyl)-3,7-dioxabicyclo[3,3,0]-Octane $((+)$-SC2) (Nakai 
et al. 2003) were prepared by the previously reported procedure (Urata et al. 2008). Piperitol ((+)-SC1m) and its positional isomer, $(+)$-SC1mr, and $(+)$-pinoresinol, and its positional isomers, $(+)$-pinoresinol $\mathrm{mrl}(\operatorname{Prmrl})$ and $(+)$-pinoresinol mr2 (Prmr2) were obtained by the reaction of $(+)$-sesamin with diisobutylalminum hydride in toluene at reflux. (+)and $(-)$-syringaresinol or $(+)$ - and $(-)$-pinoresinol were prepared by the separation of synthesized $d l$-syringaresinol or $d l$-pinoresinol (Vermes et al. 1991) in the high performance liquid chromatography (HPLC) using the chiral column. $(+)$-Pinoresinol monomethylether was prepared by hydrolysis of $(+)$-pinoresinol monomethylether monoglucoside isolated from leaves of Forsythia. (+)-Kobusin was synthesized by enzymatic reaction of recombinant CYP81Q1 proteins with $(+)$-pinoresinol monomethylether prepared from Forsythia plants. (+)-Lariciresinol and (-)-matairesinol were purchased from Arbo Nova (Finland). The other compounds were described in previously works (Noguchi et al. 2008; Ono et al. 2006).

\section{Preparation of recombinant CYP8101 proteins in Baculovirus/Sf-9 system}

Gateway system was used to construct a plasmid for heterologous expression in Sf9/Baculovirus system kit (Life technology, CA). The cDNA of CYP81Q1 was amplified by PCR using the following two primers: $5^{\prime}$ GGG GAC AAG TTT GTA CAA AAA AGC AGG CTA TG GAA GCT GAA ATGCTA TAT TCA-3' (an attB1 site is underlined) and 5'-GGG GAC CAC TTT GTA CAA GAA A GC TGG GTT CA AAC GTT GGA AAC CTG ACG AAG AA3' (an attB2 site is underlined), and then subcloned into pENTR-D-TOPO (entry vector). The subcloned fragment was sequenced to confirm the absence of PCR errors. The cDNA subcloned in the entry vector was transferred in attRcontaining destination vector, pDEST8 by LR recombination reaction.

In vitro mutagenesis of the CYP81Q1 gene was performed by recombinant PCR (Higuchi 1990) to obtain the following site-directed mutants: CYP81Q1-A308S and CYP81Q1-A308T, which have amino acid substitutions at Ala-308 replaced with serine and threonine, respectively. The primers used for PCR were as follows: CYP81Q1_full_Fw (5'-ATG GAA GCT GAA ATG CTA TAT TCA GCTCT) and CYP81Q1_full_Rv (5' - TCA AAC GTT GGA AAC CTG ACG AAGAA) for amplification of full length of CYP81Q1 and its mutants. CYP81Q1_A308S_Fw (5' -ATT GCG GGA ACG GAT TCA TCG GTT GTA ACT) and CYP81Q1_A308S_Rv (5' - AGT TACAACCGA TGA ATCCGT TCCCGCAAT) for A308S mutagenesis. CYP81Q1_A308T_Fw (5' -ATT GCG GGA ACG GAT ACA TCG GTT GTA ACT) and CYP81Q1_A308T_Rv (5'-AGT TAC AACCGA TGT ATCCGT TCC CGC AAT) for A308T mutagenesis. The sequence of the $\mathrm{Rv}$ primer for PCR-mutagenesis was complementary to that of the corresponding Fw primer. The first PCR was performed with the plasmid pENTR-D-TOPO containing cDNA of CYP81Q1 (see above) as a template using full_Fw primer and A308S-Rv, A308S-Fw and full_Rv primer, full_Fw primer and A308T-Rv, or A308T-Fw and full_Rv primer as primers, yielding A308S-a, A308S-b, A308T-a or A308T-b, respectively. The second PCR was performed with mixture of A308S-a and A308S-b or A308T-a and A308T-b as a template using full_Fw primer and full_Rv primer as primers. The amplified fragments were subcloned in pENTR-D-TOPO as described above and sequenced to confirm the absence of PCR errors. The resultant CYP81Q1-A308S and CYP81Q1-A308T in entry vector were transferred to pDEST8 as described above. Three kinds of destination vectors (CYP81Q1-WT, A308S, A308T) were transformed to E. coli (strain DH10Bac, Life technology, CA). Preparation of the recombinant CYP81Q1-baculovirus and transfection to Sf9 cells (Spodoptera frugiperda 9) were performed according to the instructions of the manufacturer (Life technology, CA). Heterologous expression of CYP81Q proteins in Sf9 cells and spectrophotometric analysis were performed as described (Saito et al. 2004).

\section{Enzyme assays and kinetics}

Enzyme assay procedure and HPLC analysis of reaction mixtures were described in our previous works (Noguchi et al. 2008; Ono et al. 2006). For relative inhibition assay, $100 \mu \mathrm{M}$ of (+)-piperitol and tested lignans were used as substrate and inhibitor, respectively. Chemical-structure confirmation of the product in the reaction of CYP81Q1 with (+)-pinoresinol monomethylether was performed by a high-performance liquid chromatography (20A series of Prominence) coupled to ion trap time-of-flight mass spectrometry (LC-IT-TOF-MS, Shimadzu, Japan) with a Develosil C30-UG-3 column $(2.0 \mathrm{~mm}$ i.d. $\times 150 \mathrm{~mm}$, Nomura Chemical, Japan). In LC conditions, Samples were eluted through the column with a gradient of water and acetonitrile containing $0.1 \%$ formic acid $(0 \mathrm{~min}$, $65: 35 ; 20 \mathrm{~min}, 0: 100 ; 27 \mathrm{~min}, 0: 100 ; 27.1 \mathrm{~min}, 65: 35 ; 38 \mathrm{~min}$, $65: 35)$. IT-TOF-MS measurements with an electrospray ionization (ESI) source in positive mode were carried out under the following conditions. The voltages of interface, skimmer, and detector were $4.5 \mathrm{kV}, 6.5 \mathrm{kV}$, and $1.68 \mathrm{kV}$. The scan range was $m / z$ 300-1000. The CDL and heat block temperatures were $200^{\circ} \mathrm{C}$. Liquid nigtrogen (purity $>99.999 \%$ ) was used as the source of nebulizer gas and drying gas. Enzyme-reaction products were detected by the corresponded ions such as a protonated ion and a dehydrated ion.

The initial velocity assays for CYP81Q1 and its mutants were carried out under steady-state conditions using the standard assay system (see above) with varying concentrations of substrates. The kinetic parameters and their standard errors were determined at nine substrate concentrations (2$100 \mu \mathrm{M})$ by fitting the initial velocity data to the MichaelisMenten equation by means of a non-linear regression analysis (Leatherbarrow 1990). Inhibition by (+)-SC1 mr was kinetically analyzed under steady-state conditions using the standard assay system (see above) with $20 \mu \mathrm{M}$ of (+)-SC1mr. The data were fitted to the competitive inhibition equation (Eq. 1) using leastsquares analysis. 


$$
1 / v=1 /\left(V_{\max }\right)+K_{\mathrm{m}}\left\{1+\left([\mathrm{I}] / K_{i}\right)\right\} /\left(V_{\max }[\mathrm{S}]\right)
$$

where $v$ and $V_{\max }$ are the initial velocity and the maximum velocity, respectively, $K_{\mathrm{m}}$ and $K_{\mathrm{i}}$ are the Michaelis constant and inhibition constant for $(+)$-SC1mr, respectively, and [S] and [I] denote concentrations of (+)-piperitol and (+)-SC1mr, respectively.

\section{Homology modeling}

The three-dimensional structural model of CYP81Q1 was constructed with homology modeling module within Insight II (Accelrys, CA) using the crystal structure data of P450 BM3 (CYP102A1, pdb code: 1bu7) as a template.The crystal structure had two water molecules near the active site of the monooxygenation reaction, thus two water molecules were placed in the same position of the initially constructed model of CYP81Q1. The water molecule 2 (w2) has been known as one of important sites for the monooxygenation reaction. The water molecule 1 (w1) placed near both w2 and the oxygen atom in the iron(IV)oxo species. We predicted that w1 was important species for MDB formation because of replacement of conserved Thr residue to Ala in CYP81Q1. The substrate, (+)-pinoresinol, was then docked into the substratebinding space near the catalytic center. The constructed (+)-pinoresinol-bound CYP81Q1 was energy-minimized with molecular mechanics, followed by optimization with molecular dynamics using Discover 3 (Accelrys, CA). To construct the model structures of $(+)$-pinoresinol-bound CYP81Q1 mutants, CYP81Q1-A308S and CYP81Q1-A308T, Ala308 of (+)-pinoresinol-bound CYP81Q1 was replaced to Ser or Thr. The initial mutant models were optimized by the same procedure as that of (+)-pinoresinol-bound CYP81Q1 to generate the structures of the mutant models, CYP81Q1-A308S and CYP81Q1-A308T.

\section{Molecular cloning and RT-PCR of CYP81038}

Phryma leptostachya naturally grown in Rokko Mountain in Kobe, Hyogo prefecture was collected in summer 2007. Total RNAs were extracted from each organ (roots, leaves, stem, and flowers) with RNeasy Plant Mini kit (QIAGEN, CA), and then reverse-transcribed into cDNA with SuperScript III (Invitrogen, CA) as described previously (Noguchi et al. 2009). A cDNA fragment responsible for CYP81Q38 was amplified by RT-PCR with the CYP81 primers of CYP81Q-Fw (5' -ATG GAA GCT GAA ATG CTA TAT TCA) and CYP81QRv (5' -TCA AAC GTT GGA AAC CTG ACG AAG AA) on cDNAs prepared from roots. The PCR with ExTaq DNA polymerase (TaKaRa Bio, Japan) was run at $94^{\circ} \mathrm{C}$ for $3 \mathrm{~min}$ followed by 28 cycles at $94^{\circ} \mathrm{C}$ for $1 \mathrm{~min}$, at $54^{\circ} \mathrm{C}$ for $1 \mathrm{~min}$ and at $72^{\circ} \mathrm{C}$ for $2 \mathrm{~min}$. Phyrma $5.8 \mathrm{~S}$ rRNA (accession DQ533822) was amplified with the primers of Pl_rRNA-Fw (5' AGG ATC ATT GTCGAA ACC TGCAA-3') and Pl_rRNA-Rv (5'-GCG CGT CGT TGG ACA CGCCGT T-3').
Lignan biotransformation by yeast cells expressing CYP81038

cDNA of CYP81Q38 was subcloned in the yeast expression vector, pYE22m as described previously (Ono et al. 2006). The resulting vector for CYP81Q38 expression, termed pYE22m-PlCYP81Q38, was used to transform a yeast strain, INVsc (Invitrogen, CA) by conventional method as described previously (Ono et al. 2006). $100 \mu \mathrm{l}$ of saturated liquid cultures of yeast cells harboring pYE22m-PlCYP81Q38 were transferred to $3 \mathrm{ml}$ of fresh medium supplemented with $100 \mu \mathrm{M}$ of (+)-piperitol. After $48 \mathrm{~h}$ of culture at $30^{\circ} \mathrm{C}$, the cultures were mixed with equal volume of methanol (final $50 \%$ methanol), sonicated together with the medium and centrifuged at $21,000 \times g$ for $10 \mathrm{~min}$ at $4^{\circ} \mathrm{C}$. The supernatant was filtered and subjected to HPLC analysis using a Develosil C30-UG-5 column (Nomura Chemical, Japan) with gradient elution program starting from $15 \%$ acetonitrile with $0.1 \%$ trifluoroacetic acid (TFA) to $80 \%$ acetonitrile with $0.1 \%$ TFA in $8 \mathrm{~min}$, followed by isocratic elution for $7 \mathrm{~min}$ at a flow rate of $1 \mathrm{ml} / \mathrm{min}$. The peaks were detected using 2996 PDA Detector (Waters, MA) at $283 \mathrm{~nm}$. The UV spectrum and the retention time $(12 \mathrm{~min})$ of the product peak were identical to those of $(+)$-sesamin standard. Yeast cells transformed with the empty vector pYE22 $\mathrm{m}$ was used as a negative control. The identity of the product was further confirmed by co-chromatography analysis by mixing $(+)$-sesamin standard with the extracts.

\section{Phylogenetic analysis}

The amino acid sequences of CYP81Qs and CYP719As were aligned by taking consideration of codon position using ClustalW bundled in SEAVIEW 4.2.4 (Gouy et al. 2010; Thompson et al. 1994). All positions containing gaps and missing data were eliminated from the further analysis. The unrooted phylogenetic trees of them were reconstructed by neighbor-joining methods from translated amino acid sequences. The alignment used to generate the tree shown in Figure 5C is available as Supplemental Data Set. The neighborjoining tree was reconstructed by SEAVIEW with the matrix of the evolutionary distances calculated by Poisson correction for the multiple substitutions. The reliability of reconstructed tree was evaluated by bootstrap test with 1000 replicates.

\section{Results}

\section{Heterologous expression of CYP8101}

After SDS-PAGE of microsomal preparation from Sf9 cells infected with baculovirus expressing CYP81Q1, whole proteins were stained with CBB. A major band was detected around $50 \mathrm{kDa}$, which is consistent with the estimated molecular weight of CYP81Q1. Moreover reduced $\mathrm{CO}$-difference spectra of the microsomal preparation clearly showed a sharp absorbance at $450 \mathrm{~nm}$ (Figure 3A). Enzyme activity assays of the microsome fraction expressing CYP81Q1 using (+)-pinoresinol and NADPH as substrate and co-substrate, respectively, 


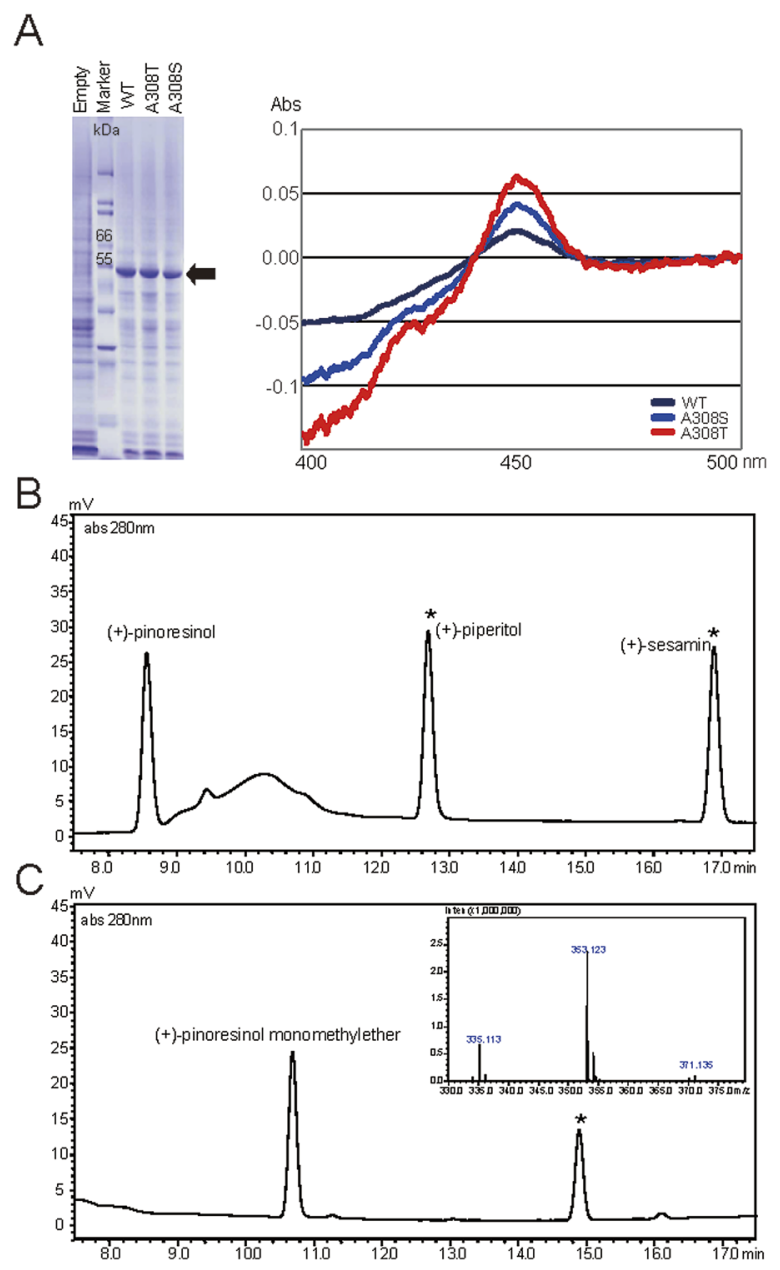

Figure 3. Heterologous expression of recombinant CYP81Q1 proteins. (A, left) SDS-PAGE of each recombinant CYP81Q1 proteins (arrow) (A, right) Reduced CO-difference spectra of microsomal preparations from Sf9 cells expressing CYP81Q1 and its mutants. Black, blue and red line indicates the spectra of CYP81Q1 (WT), A308S mutant, and A308T mutant, respectively. (B) A chromatogram at $280 \mathrm{~nm}$ absorption of reaction mixture of recombinant CYP81Q1 (WT) with (+)-pinoresinol at retention time $8.6 \mathrm{~min}$. Two asterisks indicate the products of $(+)$-piperitol at retention time $12.8 \mathrm{~min}$ and $(+)$-sesamin at retention time $16.9 \mathrm{~min}$, respectively. (C) A chromatogram at $280 \mathrm{~nm}$ absorption of reaction mixture of recombinant CYP81Q1 (WT) with (+)-pinoresinol monomethylether at retention time $10.7 \mathrm{~min}$. Asterisk indicates the product at retention time $14.9 \mathrm{~min}$. Inset indicates the MS chromatograph of the product.

resulted in the production of $(+)$-sesamin and $(+)$-piperitol (Figure 3B). These data clearly indicate that recombinant CYP81Q1 protein expressed in the baculovirus/Sf-9 cells system is functionally active.

\section{Substrate specificity of CYP8101}

We previously showed that CYP81Q1 specifically utilized $(+)$-pinoresinol and $(+)$-piperitol as substrates but did not accept $(+)$-phylligenin, $(+)$-epipinoresinol, $(+)$-pinoresinol 4'-O-glucoside or (+)-sesamolinol (Ono et al. 2006). To further investigate substrate specificity of CYP81Q1 in detail, seven naturally occurring lignans, (+)-pinoreinol monomethylether,
Table 1. Relative Inhibitory activity for MDB formation by CYP81Q1. MDB formation activity for (+)-piperitol in the absence of other lignans was set as $100 \%$.

\begin{tabular}{lcc}
\hline \multicolumn{1}{c}{ CYP81Q1(WT_A308) } & Relative activity $(\%)$ & $K_{\mathrm{i}}(\mu \mathrm{M})$ \\
\hline (+)-Piperitol & 100 & \\
(+)-Epipinoresinol & 97 & \\
(-)-Pinoresinol & 94 & \\
(+)-Pinoresinol mr2 (Pmr2) & 79 & \\
(+)-Pinoresinol mr1 (Pmr1) & 69 & \\
(+)-Kobusin & 69 & $19.8 \pm 3.5$ \\
(+)-Sclmr & 56 & \\
\hline
\end{tabular}

(+)-kobusin, (+)-syringaresinol, (-)-syringaresinol, $(-)$-lariciresinol, (-)-pinoresinol, (-)-matairesinol and an artificial synthesized lignan, $(+)-\mathrm{SC} 1 \mathrm{mr}$ were prepared and tested. In the reaction with $(+)$-pinoreinol monomethylether, CYP81Q1 produced a new product at retention time $14.9 \mathrm{~min}$ (Figure $3 \mathrm{C}$ ), which was determined to be $(+)$-kobusin with $[\mathrm{M}+\mathrm{H}]^{+}=371$ and $\left[\mathrm{M}+\mathrm{H}-\mathrm{H}_{2} \mathrm{O}\right]^{+}=353$ by LC-IT-TOF-MS. In contrast, none of the other lignans were served as substrates for CYP81Q1 in the presence of NADPH, confirming its specific substrate recognition for the same configuration to $(+)$-sesamin.

To gain insights of molecular mechanism in substrate recognition by CYP81Q1, some of these lignans were further tested as inhibitors for MDB formation from (+)-piperitol to $(+)$-sesamin by CYP81Q1 that was set as $100 \%$ activity in the absence of inhibitory chemicals (Figure 2). Neither (+)-epipinoresinol nor (-)-pinoresinol showed apparent inhibitory effects on MDB formation with the relative activity of $97 \%$ and $94 \%$, respectively, whereas (+)-kobusin and $(+)$-SC1mr showed significant inhibitory effects (relative activity of $69 \%$ and $56 \%$, respectively) (Table 1). The kinetic parameter of $(+)$-SC1mr with the highest inhibition (relative activity of 56\%) was determined to be $K_{\mathrm{i}}=19.8 \mu \mathrm{M}$. These results suggest that (+)-kobusin and (+)-SC1mr are accessible to the substrate pocket of CYP81Q1, by which MDB formation for the bona fide substrate, $(+)$-piepritol is inhibited (block out), whereas $(+)$-epipinoresinol and $(-)$-pinoresinol are not structurally accessible to the substrate pocket of CYP81Q1 due to their differential configuration.

To confirm this hypothesis, two additional synthetic lignans, (+)-pinoresinol-mr1 ((+)-Prmr1) and $(+)$-pinoresinol-mr2 $((+)-\mathrm{Prmr} 2)$ were further tested for the inhibition assay. As expected, $(+)$-Prmr1 and $(+)$-Prmr2 showed relative high inhibition activity (relative activity of $69 \%$ and $79 \%$, respectively)(Table 1). Collectively these results show that CYP81Q1 strictly discriminates substrates; $(+)$ epi-mer and $(-)$-enantiomer. In addition, the results using $(+)$ Prmr1 and (+)-Prmr2 also demonstrate that CYP81Q1 discriminates the position of the two functional groups 

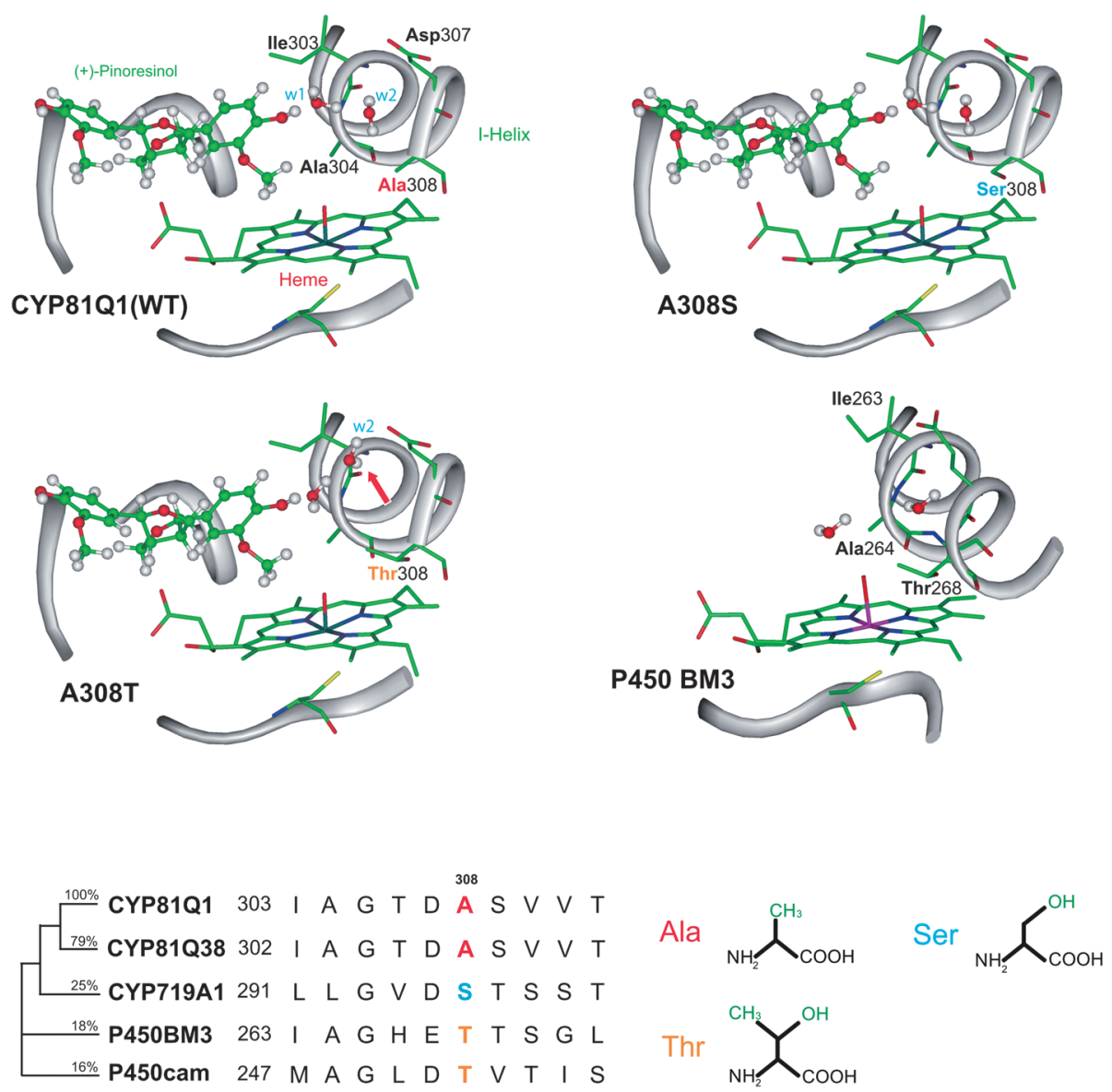

Figure 4. Homology models of CYP81Q1 proteins. These modeling structures docking with the substrate, $(+)$-pinoresinol were constructed by Insight II (Accelrys) using the crystal structure data of P450 BM3 (CYP102A1, pdb code: 1bu7) as a template. The (+)-pinoresinol-bound CYP81Q1 (WT) complex structure indicated that two water molecules (w1 and w2) appear to form a hydrogen bonding network to 4-hydroxy group of the substrate. (lower left) Alignments around distal-Thr residue in I-helix, (lower right) Side chain differences in three amino acids (Ala, Ser, and Thr).

(a hydroxy group and a methoxy group) on the aromatic ring of substrates, further establishing the substrate selectivity of CYP81Q1.

\section{Homology modeling of CYP81Q1}

As previously indicated, MDB forming P450s do not possess conserved Thr residue in the distal I helix that is considered to be crucial for $\mathrm{O}-\mathrm{O}$ bond scission in the P450 reaction cycle (Imai et al. 1989; Ono et al. 2006). However, all CYP81Qs and CYP719As that have been identified so far have Ala and Ser residues, respectively, at the corresponding position in the distal I helix, suggesting that the Thr residue conserved among other P450s is not indispensable for MDB formation (Figure 4). In turn, Ala or Ser residue observed in MDB forming P450s are likely to be involved in the catalyses for MDB formation.

To gain structural insights into MDB formation by P450s, homology models of CYP81Q1 were constructed using the crystal structure data of P450 BM3 as a template. The putative (+)-pinoresinol-bound CYP81Q1 (WT) complex structure elucidated by homology modeling indicated that two water molecules (w1 and w2) appear to form hydrogen bonding networks to 4-hydroxy group of $(+)$-pinoresinol (Figure 4$)$. The w2 is able to interact with the two carbonyl groups of the main chain of Ile303 and Ala304. These data suggest that Ala304 together with Ile303 is indispensable for the reaction.

On the other hand, the significance of Ser308 in the catalysis was examined by constructing a homology model of A308S in which Ala308 was replaced with Ser residue. The result demonstrated that Ser residue was located close to the w2 molecule but the position of w2 was analogous to that in WT. In contrast, in the model of A308T in which Ala308 was replaced with Thr residue, Thr was predicted to drastically hamper the position of the w2. These homology models support the notion that amino acid residue on the position responsible to conserved distal-Thr residue is involved in MDB formation.

\section{Site-directed mutagenesis of CYP8101}

To assess significance of the Ala308 residue for MDB formation biochemically, the two amino acid substitutions A308S and A308T were introduced into 
CYP81Q1 (WT), and heterologously expressed in Sf9 cells. CBB staining and CO-difference spectra of the mutant proteins confirmed that these CYP81Q1 mutant proteins are functionally expressed (Figure 3). Using these recombinant proteins, kinetic parameters were determined. As shown in Table 2, CYP81Q1-A308S exhibited comparable substrate specificity $\left(K_{\mathrm{m}}\right)$ to those of WT, and its catalytic activity $\left(\mathrm{k}_{\mathrm{cat}}\right)$ was moderately decreased to approximately one-third of WT.

In sharp contrast, both $K_{\mathrm{m}}$ and $\mathrm{k}_{\text {cat }}$ of CYP81Q1A308T were significantly altered. Compared to those of WT, the $K_{\mathrm{m}}$ was increased to 6 -fold and the $\mathrm{k}_{\text {cat }}$ was

Table 2. Kinetic analysis of CYP81Q1 and the mutant proteins.

\begin{tabular}{lccc}
\hline CYP81Q1 & $K_{\mathrm{m}}(\mu \mathrm{M})$ & $\mathrm{k}_{\text {cat }}\left(\mathrm{S}^{-1}\right)$ & $\mathrm{k}_{\text {cat }} / K_{\mathrm{m}}\left(\mathrm{S}^{-1} / \mu \mathrm{M}\right)$ \\
\hline WT_A308 & $1.98 \pm 0.30$ & $0.173 \pm 0.004$ & 0.087 \\
A308S & $1.96 \pm 0.58$ & $0.051 \pm 0.002$ & 0.026 \\
A308T & $13.33 \pm 3.90$ & $0.0033 \pm 0.0003$ & 0.00025 \\
\hline
\end{tabular}

decreased to approximately 52 -fold (Table 2 ). These results demonstrate that a single amino acid substitution of Ala residue by $\mathrm{Thr}$ residue severely causes catalytic impairment in MDB formation, unlike another substitution by Ser residue, thus support the notion that amino acid residue responsible for the conserved distal-Thr residue is involved in MDB formation. These data showed that amino acid residue on the position responsible to conserved distal-Thr residue is crucial for MDB formation.

\section{Identification of Phryma leptostachya CYP81038}

Phryma leptostachya (Phrymaceae) is a lignan-producing plant, which belongs to Lamiales, and is a close relative to Sesamum plants (Pedaliaceae) (Figure 5A). Since $P$. leptostachya is known to accumulate phrymarolin I and other characteristic furofuran lignans with MDB in the roots (Figure 1) (Taniguchi and Oshima 1972), enzyme genes involved in the formation of MDB of
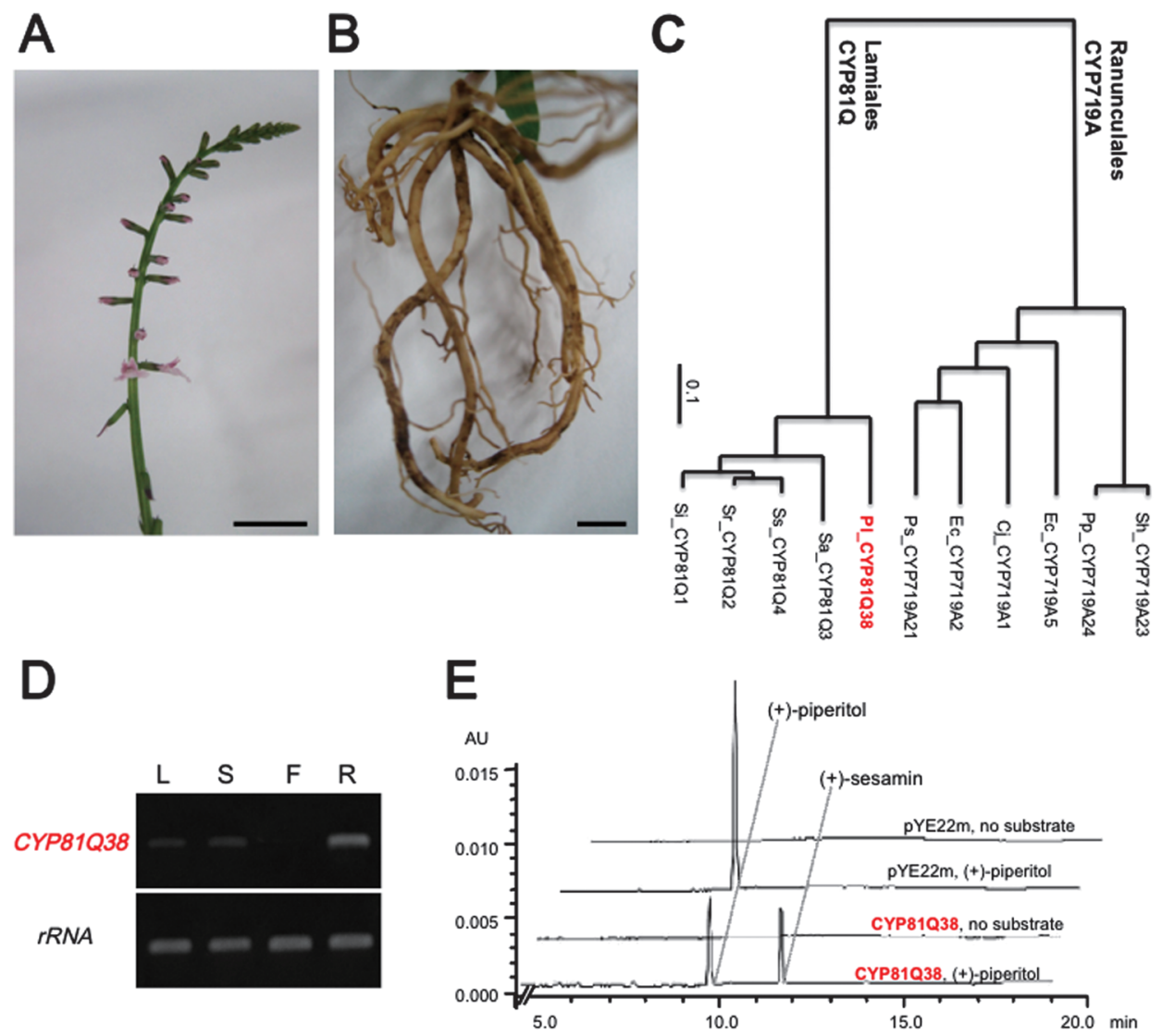

Figure 5. Characterization of Phryma leptostachya CYP81Q38. Inflorescence (A) and roots (B) of P. leptostachya, Size bar in photos indicates $1 \mathrm{~cm}$. (C) A phylogenetic tree of MDB-forming P450s. An unrooted phylogenetic tree was constructed as described in Materials and methods. The alignment used for this analysis is available as Supplemental Data Set. The percentages of replicate trees, in which the associated taxa clustered together in the bootstrap test (1000 replicates), are shown. Bar $=0.1$ amino acid substitutions per site. $\mathrm{Si}, \mathrm{Sr}, \mathrm{Ss}, \mathrm{Sa}, \mathrm{Pl}, \mathrm{Ps}, \mathrm{Ec}, \mathrm{Cj}, \mathrm{Pp}$, and Ph indicates Sesamum indicum, Sesamum radiatum, Sesamum schinzianum, Sesamum alatum, Phryma leptostachy, Papaver somniferum, Eschscholzia californica, Coptis japonica, Podophyllum peltatum, and P. hexandrum, respectively. (D) Gene expression analysis of CYP81Q38 in separated organs by RT-PCR. L: leaf, S: stem, F: flower, R: root (E) CYP81Q38 catalyzes the conversion of piperitol to sesamin. $3 \mathrm{ml}$ cultures of yeast cells expressing CYP81Q38 were supplemented with $100 \mu \mathrm{M}(+)$-piperitol $24 \mathrm{~h}$ after the subculturing, and were further cultured for $48 \mathrm{~h}$ at $30^{\circ} \mathrm{C}$ before extracted and subjected to HPLC analysis. pYE22m, a yeast cell line harboring an empty vector pYE22m. CYP81Q38, a yeast cell line that constitutively expresses CYP81Q38 protein. Shown is the representative data of three independent experiments. 
the lignans is also expected to be expressed in the roots (Figure 5B). Based on the chemotaxonomic relationship that several lineages in Lamiales produce lignans with MDB, CYP81Q1 homologs were explored as candidates for MDB forming enzyme in P. leptostachya. A cDNA fragment with high structural similarity to CYP81Q1 was amplified by RT-PCR using cDNA prepared from the root as a template, and then designated as CYP81Q38 (79\% amino acid sequence identity with CYP81Q1; Accession number: AB923911) by $\mathrm{P} 450$ nomenclature (Nelson. 2009) (Figure 5C). CYP81Q38 also have an Ala residue (Ala307), corresponding to Ala308 in CYP81Q1, at the position of distal-Thr residue (Figure 4). Semiquantitative RT-PCR confirmed that CYP81Q38s is highly expressed in root (Figure 5D).

In order to test whether CYP81Q38 catalyzes the formation of MDB on lignans, yeast suspension cultures expressing CYP81Q38 by a constitutive GAPDH promoter were fed with possible lignans. After $48 \mathrm{~h}$ of incubation, no additional peaks of lignans other than tested substrates were observed in the yeast cultures harboring a control vector. In contrast, production of $(+)$-sesamin was detected in the CYP81Q38-expressing cultures when fed with (+)-piperitol (Figure 5E). Similarly, $(+)$-sesamin and $(+)$-piperitol were detected in the yeast when fed with $(+)$-pinoresinol (data not shown). However, CYP81Q38 did not produce any detectable products in the reaction with $(+)$-sesamolinol, $(+)$-epipinoresinol, (-)-pinoresinol or (+)-SC1mr (data not shown).

\section{Discussion}

\section{A possible mode-of-action of MDB formation catalyzed by CYP8101}

CYP81Q1 is a Sesamum indicum PSS which specifically catalyzes $(+)$-sesamin formation form $(+)$-pinoresinol via (+)-piperitol by forming two MDBs (Figures 2, $3 \mathrm{~B})$. Investigation of various natural and non-natural compounds as possible substrates for MDB formation by CYP81Q1 revealed that CYP81Q1 utilizes only $(+)$-pinoresinol monomethylether, over all other compounds tested in the study. Formation of a MDB on $(+)$-pinoresinol monomethylether by CYP81Q1 resulted in $(+)$-kobusin (Figure 3C), and CYP81Q1 was inactive for $(-)$-pinoresinol, are clearly consistent with the previous report that $(+)$-kobusin is found in sesame seeds, and only $(+)$-pinoresinol is selectively metabolized into $(+)$-piperitol and $(+)$-sesamin in the presence of $( \pm)$-pinoresinols in vivo (Kato et al. 1997). Synthetic (+)-Sc1mr, (+)-Prmr1 and (+)-Prmr2 showed inhibitory effect on MDB formation, indicating that CYP81Q1 strictly discriminates configuration and position of functional groups on the aromatic ring of the substrates. This selectivity might be responsible for optical purity of $(+)$-sesamin and its related lignans with MDB such as $(+)$-sesaminol in sesame seeds. These results strongly support that CYP81Q1 is the enzyme responsible for $(+)$-sesamin biosynthesis in vivo.

The homology modeling approach suggests that the lack of a distal-Thr generally conserved in P450s is crucial for MDB forming P450s. The negligible activity of CYP81Q1-A308T mutant and the comparable activity of CYP81Q1-A308S mutant point out an important notion that hydrogen-bonding network is involved in their catalysis. Severe defect observed in CYP81Q1A308T mutant demonstrates that the ordered hydrogenbonding network in their homology models, leading to 4-hydoroxy group of the substrate, is catalytically important for MDB formation. The Thr residue probably drastically affects the position of the w2, due to a characteristic methyl group of the Thr side chain. The results that hydroxyl group and methoxy group on aromatic ring of the substrates were not interchangeable also support this notion.

On the other hand, Ser residue in the A308S mutant model does not seem to affect this hydrogen-bonding network, which is in good accordance with the result that A308 mutant exhibited the catalytic activity at the level equivalent to that of wild type CYP81Q1 (Table 2). These results substantiate the fact that distal-Thr commonly conserved among P450s is replaced with Ser in another class of MDB forming enzymes, CYP719As. Collectively, our results suggest that $\mathrm{A} 308$ is not a catalytic residue of CYP81Q1 but rather facilitates MDB formation by providing space for putative 'catalytic' water molecules. Thus, it is plausible to conclude that the establishment of substrate-participated hydrogen-bonding network triggers an intramolecular nucleophilic cyclization to elaborate a MDB structure (Figure 6), and moreover, the substitution of amino acids proximal to the distal-Thr residue to Ala (probably also to Ser) is crucial in evolving MDB forming activity. It should be noted that P450cam T252A and T252S mutants produce $\mathrm{H}_{2} \mathrm{O}_{2}$, compared to the wild type (T252)(Imai et al. 1989). Thus, the possibility that reactive oxygen species (ROS) might be mediated in catalysis for MDB formation is not excluded.

\section{Molecular evolution of MDB forming P450s}

A novel cDNA encoding a MDB-forming P450, CYP81Q38, was isolated from root of P. leptostachya based on the sequence homology to $S$. indicum PSS, CYP81Q1. Its significant expression in roots as well as its MDB-forming activity on $(+)$-piroresinol and $(+)$-piperitol suggests that $(+)$-phrymarolin I and its-related lignans with MDB are biosynthesized via MDB-formation by CYP81Q38. The two PSS enzymes, CYP81Q38 from P. leptostachya and CYP81Q1 from $S$. indicum, are most likely derived from a common gene in their ancestral plant, considering the close phylogenic 


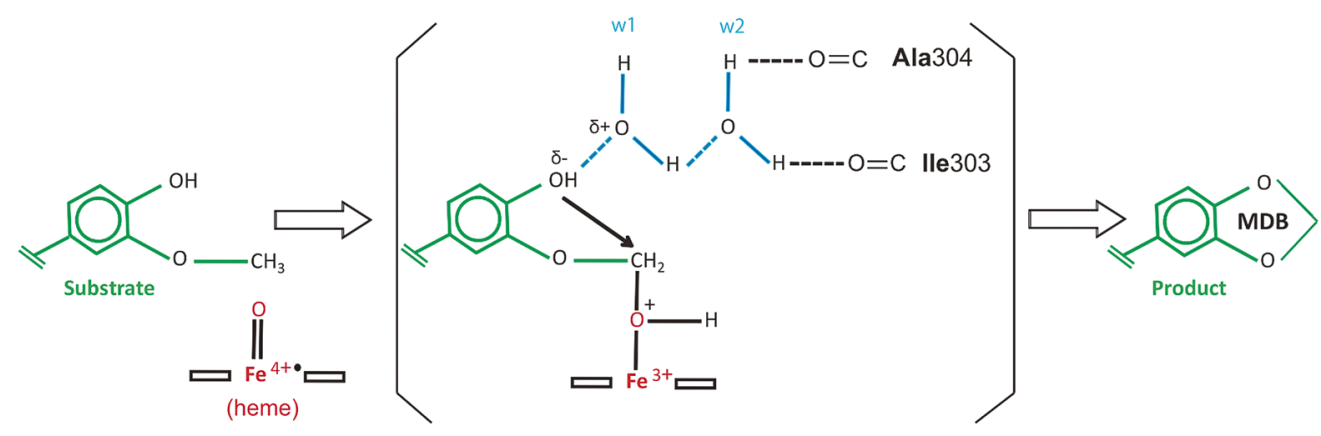

Figure 6. A possible mode-of-action of MDB formation by CYP81Q1. Based on the biochemical and modeling studies on CYP81Q1 mutants, we hypothesized a possible mechanism of MDB formation. As indicated by homology modeling, interaction of carbonyl group of the main chain of Ile303 or Ala304 with w2 potentially initiates a hydrogen bonding relay to the hydroxyl group of the substrate via two water molecules (w1, w2), resulting in activation of hydroxy group of the substrate. The activated oxygen causes nucleophilic attack (black arrow) to proximal methoxy group at the ortho-position. Cleavage of the link between the substrate and heme results in the formation of MDB on the substrate. Thus, MDB formation is considered to be a substrate-assisted tandem reaction of mono-oxygenation and nucleophilic cyclization.

relationship of $P$. leptostachya and $S$. indicum and striking overall structural similarity between CYP81Q38 and CYP81Q1 with the characteristic Ala residue at the position of distal-Thr residue, although their gene expression profiles are distinct each other (mainly in root and seed, respectively)(Figures 5C, 5D). Thus, other phylogenetically related plants producing $(+)$-sesamin and its structurally related metabolites with MDB might also have an orthologous gene responsible to PSS. In this context, a woody plant of Lamiales, Paulownia, is one candidate plant since it accumulates a large amount of $(+)$-sesamin and its derivative $(+)$-paulownin in their heartwoods often used for traditional Japanese chests (Figure 1).

It should be noted that CYP719A is also known as a class of MDB forming P450 distinct from CYP81Qs so far. CYP81Qs and CYP719As are structurally discrete as shown in Figure $5 \mathrm{C}$, and are indeed derived from phylogenetically unrelated plant lineages (Lamiales and Ranunculales order): Sesamum (Pedaliaceae) and Phryma (Phrymaceae) belong to Lamiales order, whereas Coptis japonica (Berberidaceae), opium poppy (Ranunculaceae), and Podophyllum hexandrum (Papaveraceae) belong to Ranunculales order. From the viewpoint of chemotaxonomy, Podophyllum is a unique lineage in Ranunculales because they exceptionally produce anti-tumor lignans with MDB such as podophyllotoxin instead of isoquinoline alkaloids that are more often observed specialized metabolites in Ranunculales plants. Therefore, it is conceivable that CYP719A have differentiated to be MDB forming P450 enzymes that are originally involved in the biosynthesis of isoquinoline alkaloids in the ancestral Ranunculales plant, and later Podophyllum CYP719A23 and CYP719A24 were specifically recruited to lignan biosynthesis (Marques et al. 2013). Since CYP719As commonly have a Ser residue instead of Ala residue, at the position of the conserved distal-Thr residue, it is fairly feasible that the crucial substitution events at the conserved Thr residue occurred independently in the distinct MDB forming P450s (CYP81Q and CYP719A). Furthermore, this notion is also applicable to the cases that phylogenetically unrelated lineages produce the same metabolites with MDB. For instance, Gingko (gymnosperm) and Magnolia plants (primitive angiosperm) are basal lineages of seed plants and phylogenetically distant from Lamiales. However, both plants are known to produce sesamin (Umezawa 2003a, 2003b). Furthermore, KNApSAcK database search shows that sesamin has been found in various plants more than 130 species (Afendi et al. 2012), implying that PSS have independently occurred several times during seed plant evolution. Analogously, it is also suggested that various natural products with MDB that are widespread in plants occurred as consequence of convergent evolution of MDB forming activity (Figure 1). In addition to lineagespecific local gene multiplication (Chae et al. 2014) and spatiotemporal alteration of gene expression, functional plasticity of enzyme would be a driving force to generate vast structural diversity of specialized metabolites in nature.

\section{Acknowledgements}

We thank Drs. Honoo Satake and Takashi Iwashita (SUNBOR), Atsushi Ohgaki, Asako Iuchi-Okada, Namino Tomimori, Masaaki Nakai, Yuko Fukui (SWE), Atsushi Okazawa (Osaka Pref. Univ.), Masayuki Yamamoto and Kyoji Yamada (Toyama Univ.), Naoki Shibata and Yoshiki Higuchi (Hyogo Pref. Univ.), Hideo Ohkawa (Fukuyama Univ.), Kyoko Ishiguro (Mukogawa Women's Univ.), Eiichi Mizohata and Tsuyoshi Inoue (Osaka Univ.), and Nobuhiro Ikezawa (Mitsubishi Chemicals Ltd.) for fruitful discussion and technical supports to this work.

\section{References}

Afendi FM, Okada T, Yamazaki M, Hirai-Morita A, Nakamura Y, Nakamura K, Ikeda S, Takahashi H, Altaf-Ul-Amin M, Darusman LK, et al. (2012) KNApSAcK family databases: integrated metabolite-plant species databases for multifaceted 
plant research. Plant Cell Physiol 53: e1

Apers S, Vlietinck A, Pieters L (2003) Lignans and neolignans as lead compounds. Phytochem Rev 2: 201-217

Ayres DC, Loike JD (1990) Lignans-chemical, biological, and clinical properties. In: Phillipson JD, Ayres DC, Baxter H (eds) The Origin of Sesame. Cambridge University Press, Cambridge, UK, pp 1-11

Chae L, Kim T, Nilo-Poyanco R, Rhee SY (2014) Genomic signatures of specialized metabolism in plants. Science 344 : 510-513

Gouy M, Guindon S, Gascuel O (2010) SeaView version 4: A multiplatform graphical user interface for sequence alignment and phylogenetic tree building. Mol Biol Evol 27: 221-224

Higuchi R (1990) PCR Protocols: A Guide to Methods and Applications. Academic Press, California, USA

Ikezawa N, Tanaka M, Nagayoshi M, Shinkyo R, Sakaki T, Inouye K, Sato F (2003) Molecular cloning and characterization of CYP719, a methylenedioxy bridge-forming enzyme that belongs to a novel P450 family, from cultured Coptis japonica cells. J Biol Chem 278: 38557-38565

Ikezawa N, Iwasa K, Sato F (2007) Molecular cloning and characterization of methylenedioxy bridge-forming enzymes involved in stylopine biosynthesis in Eschscholzia californica. FEBS J 274: 1019-1035

Ikezawa N, Iwasa K, Sato F (2009) CYP719A subfamily of cytochrome $\mathrm{P} 450$ oxygenases and isoquinoline alkaloid biosynthesis in Eschscholzia californica. Plant Cell Rep 28: 123-133

Imai M, Watanabe $\mathrm{Y}$, Matsushima-Hibiya $\mathrm{Y}$, Makino R, Koga $\mathrm{H}$, Horiuchi T, Ishimura Y (1989) Uncoupling of the P450cam monooxygenase reaction by a single mutation, threonine- 252 to alanine or valine: Possible role of the hydroxy amino acid in oxygen activation. Proc Natl Acad Sci USA 86: 7823-7827

Kato MJ, Chu AC, Davin LB, Lewis NG (1998) Biosynthesis of antioxidant lignans in Sesamum indicum. Phytochemistry 47: 583-591

Katsuzaki H, Kawasumi M, Kawakishi S, Osawa T (1992) Structure of novel antioxidative lignan glucosides isolated from sesame seed. Biosci Biotechnol Biochem 56: 2087-2088

Katsuzaki H, Kawakishi S, Osawa T (1994) Sesaminol glucosides in sesame seeds. Phytochemistry 35: 773-776

Leatherbarrow RJ (1990) Using linear and non-linear regression to fit biochemical data. Trends Biochem Sci 15: 455-458

MacRae WD, Towers GHN (1984) Biological activities of lignans. Phytochemistry 23: 1207-1220

Marques JV, Kim KW, Lee C, Costa MA, May GD, Crow JA, Davin LB, Lewis NG (2013) Next generation sequencing in predicting gene function in podophyllotoxin biosynthesis. J Biol Chem 288: 466-479

Moazzami AA, Kamal-Eldin A (2006) Sesame seed is a rich source of dietary lignans. J Am Oil Chem Soc 83: 719-723

Nakai M, Harada M, Nakahara K, Akimoto K, Shibata H, Miki W, Kiso Y (2003) Novel antioxidative metabolites in rat liver with ingested sesamin. J Agric Food Chem 51: 1666-1670
Nelson DR (2009) The Cytochrome P450 Homepage. Hum Genomics 4: 59-65

Noguchi A, Fukui Y, Iuchi-Okada A, Kakutani S, Satake H, Iwashita T, Nakao M, Umezawa T, Ono E (2008) Sequential glucosylation of a furofuran lignan, (+)-sesaminol, by Sesamum indicum UGT71A9 and UGT94D1 glucosyltransferases. Plant $J$ 54: 417-427

Noguchi A, Horikawa M, Fukui Y, Fukuchi-Mizutani M, IuchiOkada A, Ishiguro M, Kiso Y, Nakayama T, Ono E (2009) Local differentiation of sugar donor specificity of flavonoid glycosyltransferase in Lamiales. Plant Cell 21: 1556-1572

Ono E, Nakai M, Fukui Y, Tomimori N, Fukuchi-Mizutani M, Saito M, Satake H, Tanaka T, Katsuta M, Umezawa T, et al. (2006) Formation of two methylenedioxy bridges by a Sesamum CYP81Q protein yielding a furofuran lignan, (+)-sesamin. Proc Natl Acad Sci USA 103: 10116-10121

Osawa T, Nagata M, Namiki M, Fukuda Y (1985) Sesamolinol, an antioxidant isolated from sesame seeds. Agric Biol Chem 49: 3351-3352

Saito S, Hirai N, Matsumoto C, Ohigashi H, Ohta D, Sakata K, Mizutani M (2004) Arabidopsis CYP707As encode (b)-abscisic acid 8 '-hydroxylase, a key enzyme in the oxidative catabolism of abscisic acid. Plant Physiol 134: 1439-1449

Satake H, Ono E, Murata J (2013) Recent advances in the metabolic engineering of lignan biosynthesis pathways for the production of transgenic plant-based foods and supplements. J Agric Food Chem 61: 11721-11729

Takemura T, Ikezawa N, Iwasa K, Sato F (2013) Molecular cloning and characterization of a cytochrome P450 in sanguinarine biosynthesis from Eschscholzia californica cells. Phytochemistry 91: 100-108

Taniguchi E, Oshima Y (1972) Phrymarolin-I, a novel lignan from Phryma leptostachya L. Agric Biol Chem 36: 1013-1025

Thompson JD, Higgins DG, Gibson TJ (1994) CLUSTAL W: Improving the sensitivity of progressive multiple sequence alignment through sequence weighting, position-specific gap penalties and weight matrix choice. Nucleic Acids Res 22: 4673-4680

Umezawa T (2003a) Diversity in lignan biosynthesis. Phytochem Rev 2: 371-390

Umezawa T (2003b) Phylogenetic distribution of lignan producing plants. Wood Res 90: 27-110

Urata H, Nishioka Y, Tobashi T, Matsumura Y, Tomimori N, Ono Y, Kiso Y, Wada S (2008) First chemical synthesis of antioxidative metabolites of sesamin. Chem Pharm Bull 56: 1611-1612

Vermes B, Seligmanna O, Wagnera H (1991) Synthesis of biologically active tetrahydro-furofuranlignan-(syringin, pinoresinol)-mono- and bis-glucosides. Phytochemistry 30: 3087-3089

Winzer T, Gazda V, He Z, Kaminski F, Kern M, Larson TR, Li Y, Meade F, Teodor R, Vaistij FE, et al. (2012) A Papaver somniferum 10-gene cluster for synthesis of the anticancer alkaloid noscapine. Science 336: 1704-1708 